\title{
Risk stratification of $\mathrm{pT}$ I-3N0 patients after radical cystectomy for adjuvant chemotherapy counselling
}

\author{
E Xylinas ${ }^{1,2,13}$, EK Cha ${ }^{1,13}$, M Sun ${ }^{3}$, M Rink ${ }^{1,4}$, Q-D Trinh ${ }^{3}$, G Novara $^{5}$, DA Green', A Pycha ${ }^{6}$, Y Fradet $^{7}$, \\ S Daneshmand $^{8}$, RS Svatek', H-M Fritsche ${ }^{10}$, W Kassouf ' ', DS Scherr', T Faison', JJ Crivelli', ST Tagawa', ${ }^{12}$, \\ M Zerbib ${ }^{2}$, PI Karakiewicz ${ }^{3}$ and SF Shariat*,1,12
}

'Department of Urology, Weill Cornell Medical College, Starr 900, 525 East 68th Street, Box 94, New York, NY 10065, USA; '² Department of Urology, Cochin Hospital, APHP, Paris Descartes University, Paris, France; ${ }^{3}$ Department of Urology, University of Montreal Health Center, Montreal, QC, Canada; ${ }^{4}$ Department of Urology, University Medical Centre Hamburg-Eppendorf, Hamburg, Germany; ${ }^{5}$ Department of Urology, University of Padua, Padua, Italy; ${ }^{6}$ Department of Urology, General Hospital of Bolzano, Bolzano, Italy; ${ }^{7}$ Department of Urology, Laval University, Quebec City, QC, Canada; ${ }^{8}$ Department of Urology, University of Southern California, Los Angeles, CA, USA; ${ }^{9}$ Department of Urology, University of Texas Health Science Center, San Antonio, TX, USA; ${ }^{10}$ Department of Urology, Caritas St Josef Medical Center, University of Regensburg, Regensburg, Germany; "'Department of Urology, McGill University, Montreal, QC, Canada; '2 Division of Medical Oncology, Weill Cornell Medical College, New York, NY, USA

BACKGROUND: In PTI-T3NO urothelial carcinoma of the bladder (UCB) patients, multi-modal therapy is inconsistently recommended. The aim of the study was to develop a prognostic tool to help decision-making regarding adjuvant therapy.

METHODS: We included 2145 patients with PTI-3NO UCB after radical cystectomy (RC), naive of neoadjuvant or adjuvant therapy. The cohort was randomly split into development cohort based on the US patients $(n=1067)$ and validation cohort based on the Europe patients $(n=1078)$. Predictive accuracy was quantified using the concordance index.

RESULTS: With a median follow-up of 45 months, 5-year recurrence-free and cancer-specific survival estimates were $68 \%$ and $73 \%$, respectively. PT-stage, ge, lymphovascular invasion, and positive margin were significantly associated with both disease recurrence and cancer-specific mortality ( $P$-values $\leqslant 0.005)$. The accuracies of the multivariable models at 2,5 , and 7 years for predicting disease recurrence were $67.4 \%, 65 \%$, and $64.4 \%$, respectively. Accuracies at 2, 5, and 7 years for predicting cancer-specific mortality were 69.3\%, 66.4\%, and 65.5\%, respectively. We developed competing-risk, conditional probability nomograms. External validation revealed minor overestimation.

CONCLUSION: Despite RC, a significant number of patients with $\mathrm{PTI}$-3NO UCB experience disease recurrence and ultimately die of UCB. We developed and externally validated competing-risk, conditional probability post-RC nomograms for prediction of disease recurrence and cancer-specific mortality.

British Journal of Cancer (2012) I 07, 1826-1832. doi:I0.1038/bjc.2012.464 www.bjcancer.com

(c) 2012 Cancer Research UK

Keywords: bladder cancer; radical cystectomy; TI-3 NO; prediction; nomogram; chemotherapy

Radical cystectomy (RC) with lymphadenectomy is the standardof-care treatment for high-risk urothelial carcinoma of the bladder (UCB) (Stein et al, 2001; Shariat et al, 2006b; Hautmann et al, 2012). However, approximately $40-50 \%$ of patients experience disease recurrence within 5 years post-RC and ultimately die (Stein et al, 2001; Shariat et al, 2006b; Hautmann et al, 2012). To lower this risk, adjuvant chemotherapy has been advocated for patients with pT4 and/or lymph node positive $(\mathrm{N}+)$ disease because of their generally poor prognosis (Advanced Bladder Cancer (ABC) Meta-analysis Collaboration, 2005; von der Maase et al, 2005). Conversely, the use of adjuvant chemotherapy in patients with organ-confined and/or non-metastatic tumour invasion into perivesical soft tissue $\mathrm{UCB}$ (i.e., $\leqslant \mathrm{pT} 3 \mathrm{~N} 0$ ) remains intensely debated. This is largely due to the highly variable prognosis in this group, with up to $70 \%$ pT1-3N0 patients being cured with RC and

\footnotetext{
*Correspondence: Dr SF Shariat; E-mail: sfshariat@gmail.com

${ }^{13}$ These authors contributed equally to this work.

Received 10 July 2012; revised 4 September 2012; accepted 19 September 2012
}

bilateral lymphadenectomy (Stein et al, 2001; Shariat et al, 2006b, 2008d, 2012; Hautmann et al, 2012). Indeed, as the morbidity associated with adjuvant chemotherapy is not trivial, patients and clinicians need better risk stratification of pT1-3N0 UCB to ensure that treatment is directed toward those who need it most and are most likely to benefit from it.

To help in the clinical decision-making process of UCB patients, we and others have developed prediction tools in the hope of improving patient counselling, rational delivery of adjuvant therapies, and clinical trial design (Bochner et al, 2006; Karakiewicz et al, 2006a,b; Shariat et al, 2006a). These tools were externally validated and outperformed TNM stage-based predictions (Zaak et al, 2010; Nuhn et al, 2012). However, their utility has been hampered by the heterogeneity of the cohorts used to build the tools (various histologies Bochner et al, 2006, use of neoadjuvant and adjuvant chemotherapy (Bochner et al, 2006; Karakiewicz et al, 2006a; Shariat et al, 2006a), and failure to account for competing risks (Shariat et al, 2008b, 2009). Because of the protracted course of UCB in some patients, prediction tools may need to take competing causes of mortality into consideration to allow more precise prediction in all patients. Indeed, in pT1-3N0 
patients the risk of non-cancer-related death is high, and predictors of death overlap with predictors of the event of interest (Shariat et al, 2012). Predictions that account for competing risks are important to clinicians as well as to patients, especially when there is danger of over-treatment or sub-optimal treatment. The aim of the current study was to develop and validate prognostic tools that account for competing risks in order to facilitate clinical decision-making regarding observation vs adjuvant therapy in patients with pT1-3N0 UCB. We developed the models and validated them in European patients to ensure generalisability.

\section{MATERIAL AND METHODS}

\section{Patients}

In this institutional review board-approved study, all participating sites provided necessary institutional data-sharing agreements before the initiation of the study. A total of 10 centres worldwide provided data; none of the data from these centres were used in the original nomogram studies (Karakiewicz et al, 2006a; Shariat et al, 2006a). A computerised databank was generated for data transfer. After combining the data sets, reports were generated for each variable to identify data inconsistencies and other data integrity problems. Through regular communication with all sites, resolution of all identified anomalies was achieved before analysis. Before the final analysis, the database was frozen and the final data set was produced. From 1981 to 2008, 4160 patients underwent RC with bilateral lymphadenectomy. We excluded patients with pT0pTa-pTis disease $(n=515)$, pT4 disease $(n=372)$, lymph node metastases $(n=715)$, and those who received adjuvant chemotherapy $(n=646)$. Overall, 2145 patients with pT1-3N0 UCB, who were chemotherapy-naive, were included in the analyses.

\section{Pathological evaluation}

All surgical specimens were processed according to standard pathological procedures at each institution. Tumours were staged according to the 2002 American Joint Committee on Cancer-Union Internationale Contre le Cancer TNM classification. Tumour grade was assessed according to the $1998 \mathrm{WHO}$ /International Society of Urologic Pathology consensus classification. Lymphovascular invasion (LVI) was defined as the presence of tumour cells within an endothelium-lined space without underlying muscular walls (Shariat et al, 2010). Positive soft tissue surgical margin status (STSM) was defined as the presence of tumour at inked areas of soft tissue on the RC specimen (Novara et al, 2010).

\section{Follow-up}

Follow-up was performed according to institutional protocols. Patients were generally seen postoperatively at least every 3-4 months for the first year, semi-annually for the second year, and annually thereafter. Follow-up visits consisted of a physical examination and serum chemistry evaluation, including liver function tests and alkaline phosphatase when indicated. Diagnostic imaging of the upper tracts and chest radiography were performed at least annually or when clinically indicated. Additional radiographic evaluations were performed at the discretion of the treating physician. Disease recurrence was defined as tumour relapse in the operative field, regional lymph nodes, and/or distant metastasis; it excluded the upper tract and urethral UC occurrences. Cause of death was determined by treating physicians, by chart review corroborated by death certificates, or by death certificates alone. To reduce bias in attribution of cause of death, only patients who had bladder cancer listed in the death certificate were considered to have died of the disease for this study. All patients who were coded as having died of cancer had previous disease recurrence. Patients who died in the peri-operative period (i.e., within 30 days of surgery) were censored at time of death for cancer-specific survival analyses (Rink et al, 2012). Follow-up was defined as the time between RC and death.

\section{Statistical analyses}

The data set was split into 5 centres from North America (development cohort of 1067 patients) and 6 centres from Europe (validation cohort of 1078 patients). Recurrence-free and cancerspecific survival curves were generated using the Kaplan-Meier method. Univariable and multivariable Cox regression models addressed time to recurrence and cancer-specific mortality after RC. The full model comprised patient age, gender, pathological T-stage, grade, concomitant CIS, STSM, LVI, and the number of lymph nodes analysed. The reduced model comprised only the pathological T-stage, STSM, and LVI. We developed competingrisk nomograms for prediction of disease recurrence and cancerspecific mortality, applying conditional survival methodology (Skuladottir and Olsen, 2003; Stephenson et al, 2006). Internal and external validation of the nomogram were performed using methods described previously (Shariat et al, 2008c, 2009). The nomograms were validated in three steps. The nomogram was developed in the North American development cohort. Internal validation with 200 bootstrap resamples was applied to the multivariable Cox regression coefficients of the nomogram predictor variables to quantify their discriminant ability according to the area under the receiver-operating characteristic curve (AUC). As our data were censored, we relied on the modification of the AUC for censored data by Harell et al (1982). Under the condition of censoring, the AUC describes the probability that given two randomly drawn patients, the patient who recurs first had a higher probability of disease recurrence or cancer-specific mortality (Harell et al, 1996; Kattan et al, 1998). The second step of the nomogram validation consisted of a comparison between the nomogram-predicted and -observed probability of recurrence and cancer-specific mortality. This was plotted by using the val.surv S-Plus function devised for censored data. An ideal plot corresponds to a $45^{\circ}$ line, in which the predicted probability parallels the observed rate of recurrence and cancer-specific mortality. In the third step of the validation process, we applied the nomogram to the European validation cohort. For this external validation cohort, the nomogram-predicted probability was compared with the actual follow-up, and the AUC was calculated for specific time points after RC. All reported $P$-values are twosided and statistical significance was set at 0.05 . All analyses were performed using the S-Plus Professional software package (version 1; MathSoft Inc., Seattle, WA, USA).

\section{RESULTS}

\section{Clinico-pathological characteristics}

The clinico-pathological characteristics of the 1067 patients from the US development cohort and the 1078 patients from the European validation cohort are shown in Table 1. The patients of the development cohort had a higher proportion of concomitant CIS $(P=0.01)$ and of STSM $(P=0.02)$. Conversely, the patients of the validation cohort had a higher proportion of pT3 disease $(P=0.01)$, high-grade disease $(P=0.04)$, and LVI $(P=0.02)$. Median follow-up was longer in the development cohort than in the validation cohort $(P=0.001)$.

\section{Oncologic outcomes}

With a median follow-up of 45.2 months (mean 44.5; IQR 33.2), 581 patients $(27 \%)$ experienced disease recurrence and 456 patients $(21.2 \%)$ died from UCB. Actuarial recurrence-free survival 
Table I Clinico-pathological characteristics of 2 I 45 patients treated with RC for UCB with PTI-T3NO disease, stratified according to the development and external validation cohorts

\begin{tabular}{|c|c|c|}
\hline Characteristics & $\begin{array}{l}\text { Development cohort } \\
\quad(n=1067)\end{array}$ & $\begin{array}{l}\text { Validation cohort } \\
\quad(n=1078)\end{array}$ \\
\hline \multicolumn{3}{|l|}{ Age (years) } \\
\hline Mean & 66.2 & 65.9 \\
\hline Median & 67.3 & 66.6 \\
\hline Range & $30-91$ & $30-89$ \\
\hline \multicolumn{3}{|l|}{ Gender (n, \%) } \\
\hline Female & $229(21.5)$ & $207(19.2)$ \\
\hline Male & $838(78.5)$ & $87 \mid(80.8)$ \\
\hline \multicolumn{3}{|l|}{ Centre (n, \%) } \\
\hline । & $670(62.8)$ & \\
\hline 2 & $159(\mid 4.9)$ & \\
\hline 7 & $92(8.6)$ & - \\
\hline 8 & $51(4.8)$ & \\
\hline 11 & $95(8.9)$ & \\
\hline 3 & & $278(25.8)$ \\
\hline 6 & & $104(9.6)$ \\
\hline 9 & - & $86(8.0)$ \\
\hline 10 & & $46 \mid(42.8)$ \\
\hline 12 & & $149(13.8)$ \\
\hline \multicolumn{3}{|l|}{ Pathological T stage (n, \%) } \\
\hline TI & $295(27.6)$ & $228(21.2)$ \\
\hline $\mathrm{T} 2$ & $411(38.5)$ & $417(38.7)$ \\
\hline T3 & $361(33.8)$ & $433(40.2)$ \\
\hline \multicolumn{3}{|l|}{ Tumour grade (n, \%) } \\
\hline $1-I \mid$ & $589(55.2)$ & $298(27.6)$ \\
\hline III & $478(44.8)$ & $780(72.4)$ \\
\hline \multicolumn{3}{|l|}{ Number of nodes examined } \\
\hline Mean (median) & $26(22)$ & $19(17)$ \\
\hline Range & $0-104$ & $0-80$ \\
\hline $\operatorname{LVI}(n, \%)$ & $269(25.2)$ & $318(29.5)$ \\
\hline $\begin{array}{l}\text { Soft tissue positive surgical } \\
\text { margins }(n, \%)\end{array}$ & $44(4.1)$ & $16(1.5)$ \\
\hline Concomitant CIS (n, \%) & $545(51.1)$ & $508(47.1)$ \\
\hline Follow-up (median, IQR) & $48.7(19.7-87.7)$ & $43.8(\mid 8.8-85.1)$ \\
\hline
\end{tabular}

Abbreviations: $\mathrm{ClS}=$ carcinoma in situ; $\mathrm{IQR}=$ interquartile range $\mathrm{LVI}=$ lymphovascular invasion; $R C=$ radical cystectomy; $U C B=$ urothelial carcinoma of the bladder.

estimates at 2,5 , and 7 years after RC were $73 \% \pm 1$ (s.e.), $68 \pm 1$ and $66 \pm 1$, respectively. Actuarial cancer-specific survival estimates at 2,5 , and 7 years after RC were $82 \% \pm 1,73 \pm 1$, and $70 \pm 1$, respectively.

\section{Predictors of disease recurrence}

In univariable analyses, age, higher tumour stage (pT3), presence of grade 3, LVI, and presence of STSM were all associated with disease recurrence (Table 2). Assessment of univariable predictive accuracy (PA) estimates revealed that pT3 stage was the most informative predictor of disease recurrence $(62.3 \%)$. Presence of LVI $(60.1 \%)$ and STSM (55.2\%) were the second and third most informative predictors, respectively. In multivariable analysis that adjusted for the effects of standard clinico-pathological features, higher pathological stage (pT3), presence of LVI, and presence of STSM remained independently associated with disease recurrence (Table 2).

The full model for prediction of disease recurrence based on patient age, gender, pathological T-stage, grade, concomitant CIS, STSM, LVI, and the number of lymph nodes analysed had a $64.9 \%$,
Table 2 Univariable and multivariable Cox regression models for prediction of recurrence and predictive accuracy estimates of recurrencefree survival predictions at 2, 5, and 7 years in 2145 patients with TI-T3NO UCB treated with RC

\begin{tabular}{|c|c|c|c|c|c|c|c|}
\hline \multirow[b]{3}{*}{ Predictors } & & & & \multicolumn{4}{|c|}{ Multivariable } \\
\hline & \multicolumn{3}{|c|}{ Univariable } & \multicolumn{2}{|c|}{ Full model } & \multicolumn{2}{|c|}{$\begin{array}{c}\text { Reduced } \\
\text { model }\end{array}$} \\
\hline & HR & $P$-value & AUC & HR & $P$-value & HR & $P$-value \\
\hline \multicolumn{8}{|l|}{ Pathological T stage } \\
\hline $\mathrm{TI}^{\circ}$ & 1.00 & Reference & & 1.00 & Reference & 1.00 & Reference \\
\hline $\mathrm{T} 2$ & 1.19 & 0.3 & 62.3 & 0.98 & 0.9 & 1.06 & 0.7 \\
\hline $\mathrm{T} 3$ & 2.68 & $<0.001$ & & 1.96 & 0.003 & 2.15 & $<0.001$ \\
\hline $\begin{array}{l}\text { Lymphovascular } \\
\text { invasion }\end{array}$ & 2.56 & $<0.001$ & 60.1 & 2.18 & $<0.001$ & 2.18 & $<0.001$ \\
\hline $\begin{array}{l}\text { Positive soft tissue } \\
\text { surgical margin }\end{array}$ & 2.32 & $<0.001$ & 51.9 & 2.04 & 0.002 & 2.07 & 0.001 \\
\hline Tumour grade (III) & 1.68 & 0.002 & 55.2 & 0.92 & 0.6 & - & - \\
\hline $\begin{array}{l}\text { Number of nodes } \\
\text { (cont. coded) }\end{array}$ & 0.99 & 0.09 & 53.3 & 1.00 & 0.2 & - & - \\
\hline Concomitant CIS & 0.90 & 0.4 & 51.2 & 1.04 & 0.8 & - & - \\
\hline Age (cont. codes) & 1.01 & 0.04 & 55.2 & 1.00 & 0.5 & - & - \\
\hline Gender (male) & 1.11 & 0.5 & 50.2 & 1.16 & 0.3 & - & - \\
\hline \multicolumn{8}{|l|}{ C-index } \\
\hline $\begin{array}{l}\text { 2-Year } \\
\text { recurrence-free } \\
\text { survival }\end{array}$ & & & & & 64.9 & & 67.4 \\
\hline $\begin{array}{l}\text { 5-Year } \\
\text { recurrence-free } \\
\text { survival }\end{array}$ & - & - & - & & 61.9 & & 65.0 \\
\hline $\begin{array}{l}\text { 7-Year } \\
\text { recurrence-free } \\
\text { survival }\end{array}$ & & & & & 62.2 & & 64.4 \\
\hline
\end{tabular}

Abbreviations: $\mathrm{AUC}=$ area under the curve; $\mathrm{CIS}=$ carcinoma in situ; $\mathrm{HR}=$ hazard ratio; $R C=$ radical cystectomy; $U C B=$ urothelial carcinoma of the bladder.

$61.9 \%$, and $62.2 \% \mathrm{PA}$ for disease recurrence at 2, 5, and 7 years, respectively. The reduced model, based on only pathological T-stage, STSM, and LVI had a PA of $67.4 \%, 65 \%$, and $64.4 \%$ at 2,5 , and 7 years, respectively. Addition of LVI or STSM improved the accuracy of T-stage for prediction of disease recurrence by 1.4$3.1 \%(P \leqslant 0.03)$. The model exhibited good calibration across the spectrum of predictions in internal validation, but exhibited minimal optimism in lower range of prediction in external validation (data not shown). We developed a competing-risk, conditional probability nomogram for predicting recurrence-free survival for the reduced multivariable model (Figure 1). By calculating the total number of risk points on the nomogram, one is able to calculate the 2, 5, and 7-year probabilities of recurrencefree survival.

\section{Predictors of cancer-specific mortality}

In univariable analyses, age, higher tumour stage (pT3), presence of grade 3, LVI, and STSM were all associated with cancerspecific mortality (Table 3). Assessment of univariable PA estimates revealed that pT3 stage was the most informative predictor of cancer-specific mortality $(64.5 \%)$. Presence of LVI $(60.3 \%)$ and STSM (57.2\%) were the second and third most informative predictors, respectively. In multivariable analysis that adjusted for the effects of standard clinico-pathological features, higher pathological stage (pT3), LVI, and STSM remained independently associated with cancer-specific mortality (Table 3 ).

The full model had a $67.7 \%, 64.5 \%$, and $65 \%$ PA for cancerspecific mortality at 2,5 , and 7 years, respectively. The reduced 
A

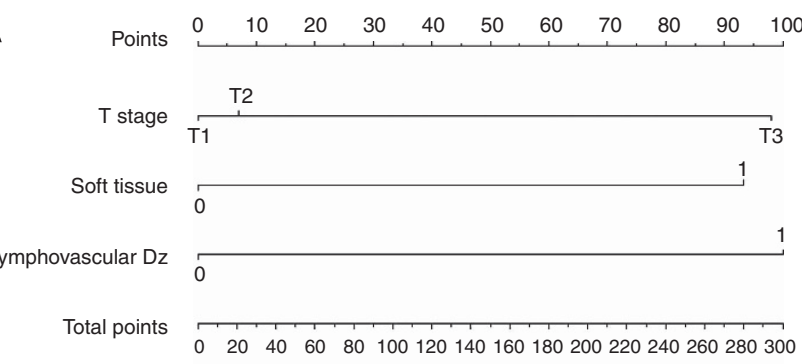

$\begin{array}{llllll}\text { 2(Y)recurrence-free } & 0.85 & 0.8 & 0.7 & 0.5 & 0.3\end{array}$

\begin{tabular}{lllll} 
5(Y)recurrence-free & & & & \\
\cline { 3 - 5 } & 0.8 & 0.7 & 0.5 & 0.3 \\
7(Y)recurrence-free & & & & \\
\cline { 3 - 6 } & 0.8 & 0.7 & 0.5 & 0.3
\end{tabular}

\section{B}
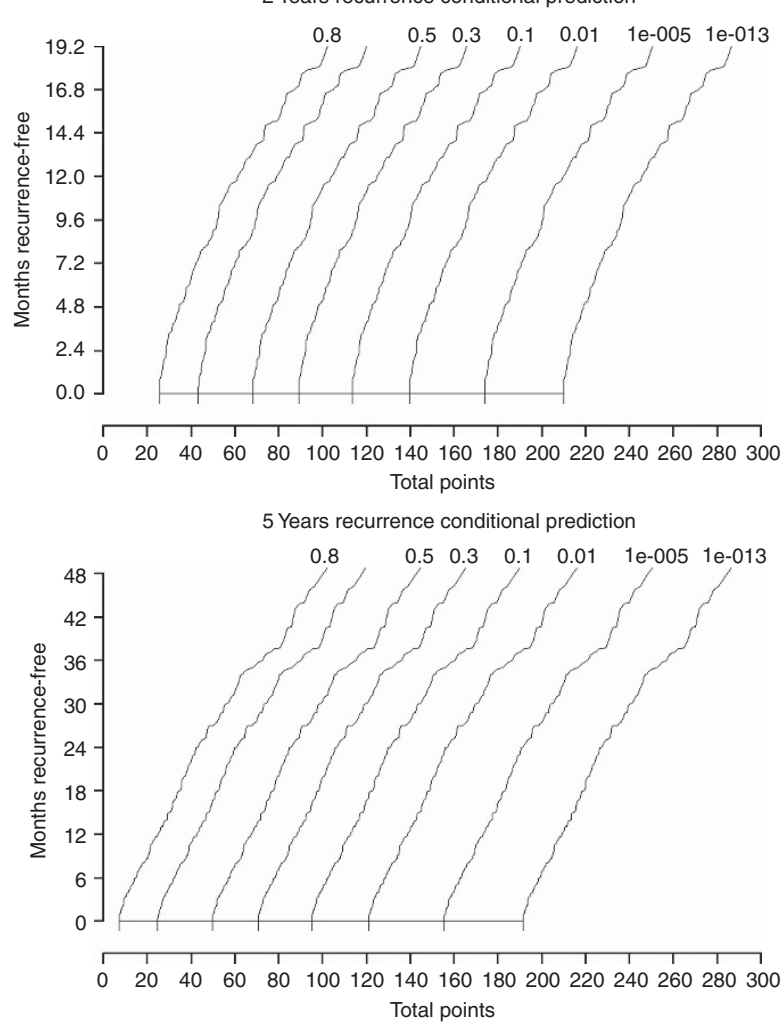

7 Years recurrence conditional prediction

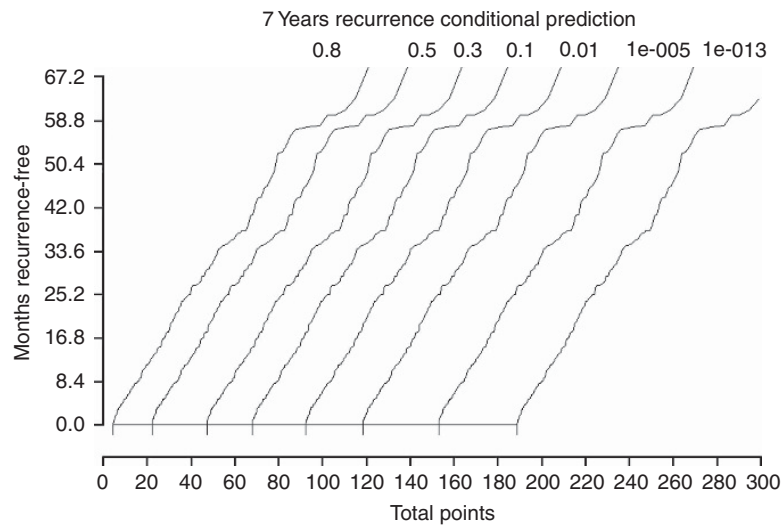

Figure I Nomogram and conditional probability predictions of recurrence-free survival at 2, 5, and 7 years after radical cystectomy.
Table 3 Univariable and multivariable Cox regression models for prediction of cancer-specific mortality and predictive accuracy estimates of CSS predictions at 2, 5, and 7 years in 2145 patients with TI-T3NO UCB treated with RC

\begin{tabular}{|c|c|c|c|c|c|c|c|}
\hline \multirow[b]{3}{*}{ Predictors } & & & & \multicolumn{4}{|c|}{ Multivariable } \\
\hline & \multicolumn{3}{|c|}{ Univariable } & \multicolumn{2}{|c|}{ Full model } & \multicolumn{2}{|c|}{$\begin{array}{c}\text { Reduced } \\
\text { model }\end{array}$} \\
\hline & HR & $P$-value & AUC & HR & $P$-value & HR & $P$-value \\
\hline \multicolumn{8}{|l|}{ Pathological T stage } \\
\hline TI & 1.00 & Reference & & 1.00 & Reference & 1.00 & Reference \\
\hline $\mathrm{T} 2$ & 1.25 & 0.2 & 64.5 & 1.00 & 1.0 & 1.11 & 0.6 \\
\hline T3 & 3.10 & $<0.001$ & & 2.22 & 0.001 & 2.49 & $<0.001$ \\
\hline $\begin{array}{l}\text { Lymphovascular } \\
\text { invasion }\end{array}$ & 2.52 & $<0.001$ & 60.3 & 2.52 & $<0.001$ & 2.07 & $<0.001$ \\
\hline $\begin{array}{l}\text { Positive soft tissue } \\
\text { surgical margin }\end{array}$ & 2.51 & $<0.001$ & 52.2 & 2.07 & 0.005 & 2.21 & 0.001 \\
\hline Tumour grade (III) & 1.63 & 0.001 & 57.2 & 0.88 & 0.5 & - & - \\
\hline $\begin{array}{l}\text { Number of nodes } \\
\text { (cont. coded) }\end{array}$ & 1.00 & 0.2 & 51.9 & 1.00 & 0.4 & - & - \\
\hline Concomitant CIS & 0.92 & 0.5 & 50.2 & 1.10 & 0.5 & - & - \\
\hline Age (cont. coded) & 1.02 & 0.004 & 57.3 & 1.01 & 0.1 & - & - \\
\hline Male gender & 1.07 & 0.7 & 50.9 & 1.11 & 0.5 & - & - \\
\hline \multicolumn{8}{|l|}{ C-index } \\
\hline 2-Year CSS & & & & & 67.7 & & 69.3 \\
\hline 5-Year CSS & - & - & - & & 64.5 & & 66.4 \\
\hline 7-Year CSS & & & & & 65.0 & & 65.5 \\
\hline
\end{tabular}

Abbreviations: $\mathrm{AUC}=$ area under the curve; $\mathrm{CIS}=$ carcinoma in situ; $\mathrm{CSS}=$ cancerspecific survival; $H R=$ hazard ratio; $R C=$ radical cystectomy; $U C B=$ urothelial carcinoma of the bladder.

model presented had a PA of $69.3 \%, 66.4 \%$, and $65.5 \%$ at 2,5 , and 7 years, respectively. Addition of LVI or STSM improved the accuracy of T-stage for prediction of cancer-specific mortality by $1.2-2.8 \%(P \leqslant 0.04)$. Similar to the recurrence prediction, the model exhibited good calibration across the spectrum of predictions in internal validation but exhibited some optimism in external validation (data not shown). We developed competingrisk, conditional probability nomograms for predicting cancerspecific survival for the reduced multivariable model (Figure 2). External validation revealed minor overestimation (Figure 3 ).

\section{DISCUSSION}

We developed postoperative nomograms, integrating competing risks, and conditional probabilities for prediction of disease recurrence and cancer-specific mortality in chemotherapy-naive pT1-3N0 patients treated with RC. Nomograms currently represent the most accurate and discriminatory tools to predict probabilities of outcomes after RC (Shariat et al, 2008d). The clinical utility (i.e., clinical decision-making regarding adjuvant chemotherapy) of previous published RC nomograms has been limited by their inclusion of all tumour stages (i.e., pT0-4, any N) of uncommon and/or extraneous histologies, and of patients receiving perioperative chemotherapy (Bochner et al, 2006; Karakiewicz et al, 2006a; Shariat et al, 2006a). To overcome these limitations, we developed nomograms that specifically address outcomes of patients with pT1-3N0 UCB, who did not receive peri-operative chemotherapy. As a significant proportion of pT1-3N0 patients are likely to die from non-cancer-related causes, we used competingrisk analyses (Cheng et al, 1998) to estimate the probability of cancer-specific mortality with higher accuracy. Our nomograms further incorporated conditional probabilities in order to allow 
A

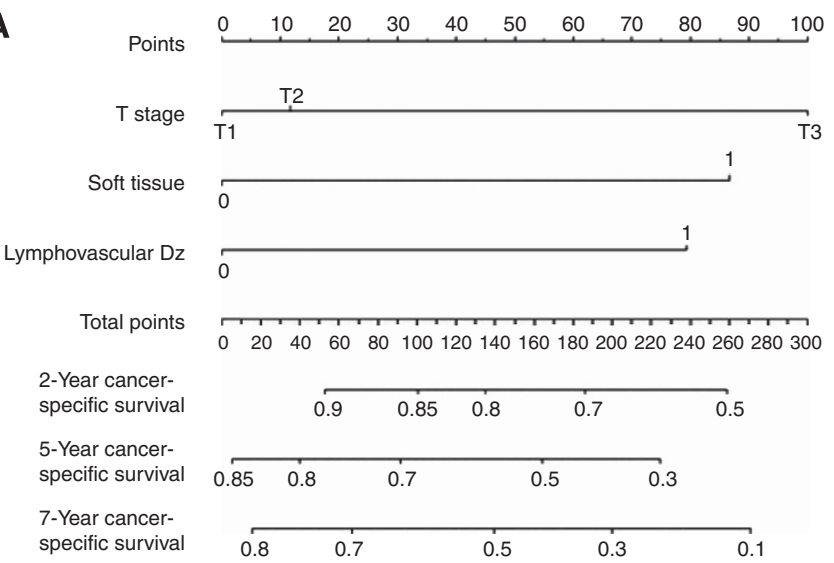

B
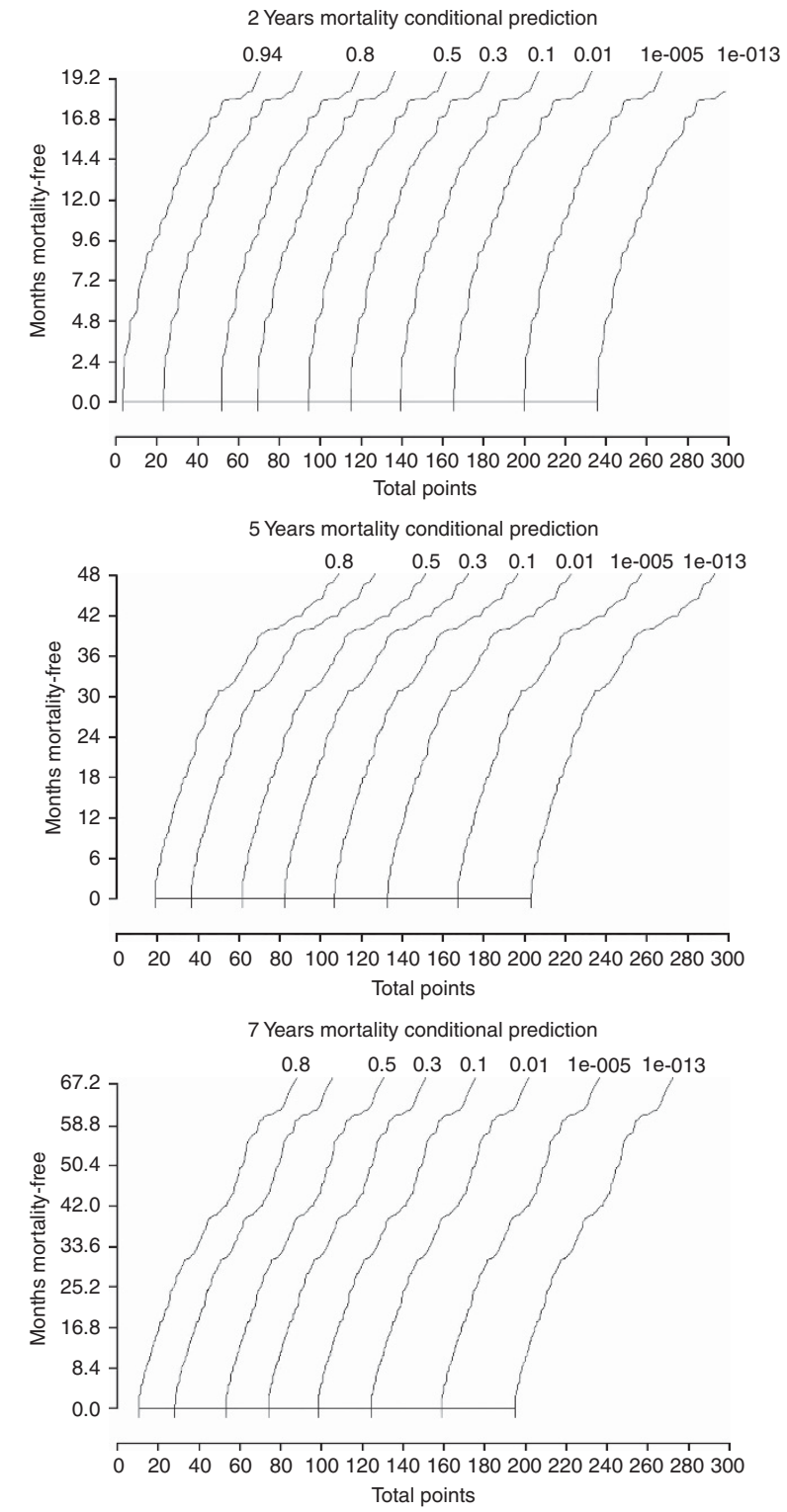

Figure 2 Nomogram and conditional probability predictions of cancerspecific survival at 2, 5, and 7 years after radical cystectomy.

accurate patient counselling not only in the immediate postoperative setting but also during the various stages of follow-up. A patient's probability of a future event changes over time; for example, a patient's risk of disease recurrence within 5 years after $\mathrm{RC}$ is higher the day after surgery than if the patient has no recurrence at 2 years. In UCB, as a patients' prognosis is expected to improve with increasing disease-free interval, absence of adjustment for this variable results in an excessively somber estimate of cancer control over time. Therefore, we provide nomograms that adjust for the effect of disease-free interval following surgery. As expected, the predicted risk of disease recurrence decreases with increasing disease-free interval.

We found that the prognosis of RC patients with pT1-3N0 UCB can be predicted with reasonable accuracy (ranging from 64-69\%). Although this rate could be considered moderate, it is within the range of performance of commonly used tools in the management of patients with prostate cancer (Shariat et al, 2008b,c). Moreover, because of the highly heterogeneous outcomes of this specific patient group, it is very difficult for a clinician to prognosticate recurrence and survival. Currently, for example, only pT3N0 patients are considered for adjuvant chemotherapy. Although T-stage represents the strongest single predictor of outcomes in pT1-3N0 patients, addition of readily available pathological variables such as LVI and STSM improved the accuracy of our models by a statistically and prognostically significant margin (Shariat et al, 2006b). Nevertheless, it is evident that even the best combination of standard clinico-pathological features is insufficient to achieve optimal prediction in pT1-3N0 UCB. Blood- and tissue-based biomarkers may represent a 'fast, easy, cheap, and powerful' method to enhance the accuracy of the current multivariable prognostic/predictive tools. We and others have shown that integration of biomarkers improves the prediction of outcomes in pT1-3N0 UCB patients by a statistically and clinically substantial margin (Gakis et al, 2011, 2012; Shariat et al, 2012). There is no doubt that panels of biomarkers that capture the biological and clinical behaviour of each individual tumour will be necessary to serve as prognosticators, predictors, therapeutic targets, and/or surrogate end points in order to usher the much awaited personalised oncology. We internally validated the performance of our nomograms in the US population it was built on, and externally in an European population (Rink et al, 2012). Differences in disease and population characteristics may undermine the discrimination and calibration of predictive tools when applied to a different population. Specific criteria used in defining the sample used to develop a prediction tool may not allow the use of tools for patients with different characteristics or who have been exposed to different treatment strategies. Indeed, there were several significant differences between the US and European pT13N0 patient cohorts (i.e., different rates of T3, high-grade, CIS, LVI, STSM). Therefore, external validation in different contemporary cohorts is necessary to ensure generalisability of prediction tools (Shariat et al, 2008b,c).

Published studies have added to general knowledge of the best candidate for adjuvant chemotherapy after RC, but physicians and patients have few tools to help them translate this body of knowledge into individualised, evidence-based recommendations. Although the power from the published randomised adjuvant chemotherapy trials remains limited (Advanced Bladder Cancer (ABC) Meta-analysis Collaboration, 2005; von der Maase et al, 2005), patients with lymph node involvement or metastatic disease are usually counselled in favour of adjuvant chemotherapy, as it has been suggested to improve disease-free survival in this population (Advanced Bladder Cancer (ABC) Meta-analysis Collaboration, 2005; von der Maase et al, 2005; Stenzl et al, 2011). In pT1-3N0 patients, the data is largely underpowered and insufficient to allow evidence-based clinical decision-making. Accurate prediction of outcomes in these patients may alleviate some of the quandary of practitioners and patients alike when faced with the potentially beneficial but toxic adjuvant therapy. Equipped with accurate prediction and a personalised clinical decision, patients are more likely to be confident in their treatment 

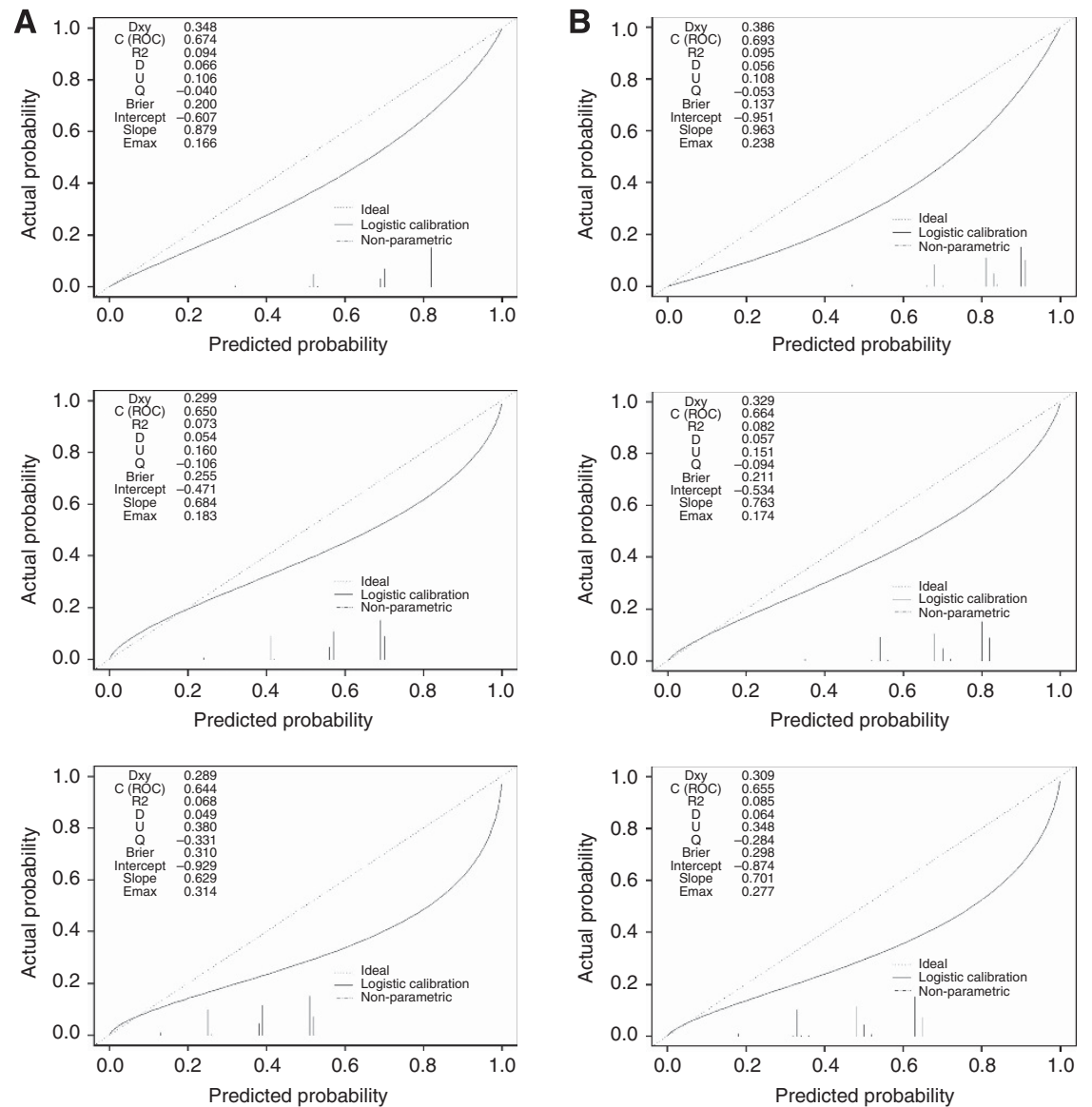

Figure 3 External validation of the nomograms for predictions of recurrence-free survival $(\mathbf{A})$ and cancer-specific survival $(\mathbf{B})$ at 2,5 , and 7 years after radical cystectomy.

decisions and less likely to experience regret in the future (Shariat et $a l, 2008 \mathrm{~b}, \mathrm{c})$. Multivariable nomograms such as the one we propose currently represent the most accurate and widely used prediction tool in oncology (Shariat et al, 2008b,c). Until better tools are available, our nomograms based on pT-stage, LVI, and STSM could help in the risk stratification of neoadjuvant chemotherapy-naive pT1-3N0 UCB patients for adjuvant chemotherapy after RC. Using decision analysis, Vickers et al (2009) recently demonstrated that nomograms can improve clinical decision-making regarding referral of patients with bladder cancer for adjuvant chemotherapy after RC. They found that nomograms outperformed current decision-making strategies based on T- and $\mathrm{N}$-stage to determine which pT1-4 any $\mathrm{N}$ bladder cancer patient would benefit from adjuvant chemotherapy, taking into account drug effectiveness and tolerability. For example, using the nomogram to identify patients with a $25 \%$ risk of disease recurrence after surgery alone as an indication for adjuvant chemotherapy reduced unnecessary treatment by approximately $25 \%$ compared with the standard approach of using pathological stage criteria.

The current study suffers from several limitations. First and foremost are the limitations inherent to the retrospective multicentre study design. The population in this study underwent RC by multiple surgeons and had specimens evaluated by multiple pathologists. However, all surgeons operated at selected tertiary care centres with significant experience in UCB management, which might increase the external validity of the data compared with the single-centre, single-surgeon setting. In addition, we did not perform a centralised pathological review, which could have led to misinterpretations of pathological specimens and underreporting. However, whereas it may be preferable for a single pathologist specialising in genitourinary pathology to review each RC specimen, the present study reflects the real-world practice. Moreover, dedicated genitourinary pathologists examined all specimens. Finally, it may be argued that the patients in our database would currently be candidates for neoadjuvant chemotherapy, thereby questioning the current applicability of our data, which were generated from patients who did not receive perioperative systemic chemotherapy. Despite the evidence regarding the efficacy of neoadjuvant chemotherapy, to date, only $9-22 \%$ of muscle-invasive UCB patients receive neoadjuvant chemotherapy before RC (Burger et al, 2012).

\section{Conclusions}

Despite radical surgery with curative intent, a significant number of patients with pT1-3N0 UCB will experience disease recurrence and, ultimately, death. We developed competing-risk, conditional probability nomograms that predict the outcomes of chemotherapy-naive pT1-3N0 UCB patients with reasonable accuracy. We internally validated the nomograms in a US population and externally validated them in a European population. Such nomograms may improve the clinical decision-making process regarding adjuvant chemotherapy and may assist in inclusion for clinical trials. 


\section{REFERENCES}

Advanced Bladder Cancer (ABC) Meta-analysis Collaboration (2005) Adjuvant chemotherapy in invasive bladder cancer: a systematic review and meta-analysis of individual patient data Advanced Bladder Cancer (ABC) Meta-analysis Collaboration. Eur Urol 48(2): 189-199

Bochner BH, Kattan MW, Vora KC (2006) Postoperative nomogram predicting risk of recurrence after radical cystectomy for bladder cancer. $J$ Clin Oncol 24(24): 3967-3972

Burger M, Mulders P, Witjes W (2012) Use of neoadjuvant chemotherapy for muscle-invasive bladder cancer is low among major european centres: results of a feasibility questionnaire. Eur Urol 61(5): 1070-1071

Cheng SC, Fine JP, Wei LJ (1998) Prediction of cumulative incidence function under the proportional hazards model. Biometrics 54(1): 219-228

Gakis G, Schwentner C, Todenhofer T, Stenzl A (2012) Current status of molecular markers for prognostication and outcome in invasive bladder cancer. BJU Int 110(2): 233-237

Gakis G, Todenhofer T, Renninger M, Schilling D, Sievert KD, Schwentner C Stenzl A (2011) Development of a new outcome prediction model in carcinoma invading the bladder based on preoperative serum C-reactive protein and standard pathological risk factors: the Tnr-C score. BJU Int 108(11): 1800-1805

Harrell Jr FE, Califf RM, Pryor DB, Lee KL, Rosati RA (1982) Evaluating the yield of medical tests. JAMA 247(18): 2543-2546

Harrell Jr FE, Lee KL, Mark DB (1996) Multivariable prognostic models: issues in developing models, evaluating assumptions and adequacy, and measuring and reducing errors. Stat Med 15(4): 361-387

Hautmann RE, de Petriconi RC, Pfeiffer C, Volkmer BG (2012) Radical cystectomy for urothelial carcinoma of the bladder without neoadjuvant or adjuvant therapy: long-term results in 1100 patients. Eur Urol 61(5): 1039-1047

Karakiewicz PI, Shariat SF, Palapattu GS, Gilad AE, Lotan Y, Rogers CG, Vazina A (2006a) Nomogram for predicting disease recurrence after radical cystectomy for transitional cell carcinoma of the bladder. J Urol 176(4 Part 1): 1354-1356

Karakiewicz PI, Shariat SF, Palapattu GS, Perrotte P, Lotan Y, Rogers CG, Amiel GE (2006b) Precystectomy nomogram for prediction of advanced bladder cancer stage. Eur Urol 50(6): 1254-1260

Kattan MW, Eastham JA, Stapleton AM, Wheeler TM, Scardino PT (1998) A preoperative nomogram for disease recurrence following radical prostatectomy for prostate cancer. J Natl Cancer Inst 90(10): 766-771

Novara G, Svatek RS, Karakiewicz PI, Skinner E, Ficarra V, Fradet Y, Lotan Y (2010) Soft tissue surgical margin status is a powerful predictor of outcomes after radical cystectomy: a multicenter study of more than 4,400 patients. J Urol 183(6): 2165-2170

Nuhn P, May M, Sun M, Fritsche HM, Brookman-May S, Buchner A, Bolenz C (2012) External validation of postoperative nomograms for prediction of all-cause mortality, cancer-specific mortality, and recurrence in patients with urothelial carcinoma of the bladder. Eur Urol 61(1): 58-64

Rink M, Fajkovic H, Cha EK, Gupta A, Karakiewicz PI, Chun FK, Lotan Y, Shariat SF (2012) Death certificates are valid for the determination of cause of death in patients with upper and lower tract urothelial carcinoma. Eur Urol 61(4): 854-855

Shariat SF, Chromecki TF, Cha EK, Karakiewicz PI, Sun M, Fradet Y, Isbarn H (2012) Risk stratification of organ confined bladder cancer after radical cystectomy using cell cycle related biomarkers. J Urol 187(2): 457-462

Shariat SF, Karakiewicz PI, Ashfaq R, Lerner SP, Palapattu GS, Cote RJ, Sagalowsky AI, Lotan Y (2008a) Multiple biomarkers improve prediction of bladder cancer recurrence and mortality in patients undergoing cystectomy. Cancer 112(2): 315-325

Shariat SF, Karakiewicz PI, Palapattu GS, Amiel GE, Lotan Y, Rogers CG, Vazina A (2006a) Nomograms provide improved accuracy for predicting survival after radical cystectomy. Clin Cancer Res 12(22): 6663-6676

Shariat SF, Karakiewicz PI, Palapattu GS, Lotan Y, Rogers CG, Amiel GE, Vazina A (2006b) Outcomes of radical cystectomy for transitional cell carcinoma of the bladder: a contemporary series from the bladder cancer research consortium. J Urol 176(6 Part 1): 2414-2422

Shariat SF, Karakiewicz PI, Roehrborn CG, Kattan MW (2008b) An updated catalog of prostate cancer predictive tools. Cancer 113(11): 3075-3099

Shariat SF, Karakiewicz PI, Suardi N, Kattan MW (2008c) Comparison of nomograms with other methods for predicting outcomes in prostate cancer: a critical analysis of the literature. Clin Cancer Res 14(14): 4400-4407

Shariat SF, Kattan MW, Vickers AJ, Karakiewicz PI, Scardino PT (2009) Critical review of prostate cancer predictive tools. Future Oncol 5(10): 1555-1584

Shariat SF, Margulis V, Lotan Y, Montorsi F, Karakiewicz PI (2008d) Nomograms for bladder cancer. Eur Urol 54(1): 41-53

Shariat SF, Svatek RS, Tilki D, Skinner E, Karakiewicz PI, Capitanio U, Bastian PJ (2010) International validation of the prognostic value of lymphovascular invasion in patients treated with radical cystectomy. BJU Int 105(10): 1402-1412

Skuladottir H, Olsen JH (2003) Conditional survival of patients with the four major histologic subgroups of lung cancer in Denmark. J Clin Oncol 21(16): 3035-3040

Stein JP, Lieskovsky G, Cote R, Groshen S, Feng AC, Boyd S, Skinner E (2001) Radical cystectomy in the treatment of invasive bladder cancer: long-term results in 1,054 patients. J Clin Oncol 19(3): 666-675

Stenzl A, Cowan NC, De Santis M, Kuczyk MA, Merseburger AS, Ribal MJ, Sherif A, Witjes JA (2011) Treatment of muscle-invasive and metastatic bladder cancer: update of the Eau guidelines. Eur Urol 59(6): 1009-1018

Stephenson AJ, Scardino PT, Eastham JA, Bianco Jr FJ, Dotan ZA, Fearn PA, Kattan MW (2006) Preoperative nomogram predicting the 10-year probability of prostate cancer recurrence after radical prostatectomy. J Natl Cancer Inst 98(10): 715-717

Vickers AJ, Cronin AM, Kattan MW, Gonen M, Scardino PT, Milowsky MI, Dalbagni G, Bochner BH (2009) Clinical benefits of a multivariate prediction model for bladder cancer: a decision analytic approach. Cancer 115(23): 5460-5469

von der Maase H, Sengelov L, Roberts JT, Ricci S, Dogliotti L, Oliver T, Moore MJ, Zimmermann A, Arning M (2005) Long-term survival results of a randomized trial comparing gemcitabine plus cisplatin, with methotrexate, vinblastine, doxorubicin, plus cisplatin in patients with bladder cancer. J Clin Oncol 23(21): 4602-4608

Zaak D, Burger M, Otto W, Bastian PJ, Denzinger S, Stief CG, Buchner H (2010) Predicting individual outcomes after radical cystectomy: an external validation of current nomograms. BJU Int 106(3): 342-348

This work is published under the standard license to publish agreement. After 12 months the work will become freely available and the license terms will switch to a Creative Commons Attribution-NonCommercial-Share Alike 3.0 Unported License. 